\title{
Posterior Circulation Stroke: Advances in Understanding and Management
}

\author{
Geoffrey A. Donnan \\ Department of Neurology, Melbourne Brain Centre, Royal Melbourne Hospital, University of Melbourne, Melbourne, Australia
}

This important contribution to the stroke literature is edited by Professor Jong S. Kim, one of the most distinguished investigators in the field posterior circulation stroke, together with many colleagues from other parts of the world. In 15 chapters and 238 pages Professor Kim has put together a superb compilation of everything you need to know about posterior circulation stroke. This is a Springer publication; my copy was in hardback with good quality paper with excellent diagrams and photographs, making it a pleasure to read (Figure 1).

Posterior circulation stroke has always appealed to neurologists because of the long history of careful clinical pathological correlation with phenotypic description. Nowhere else in the

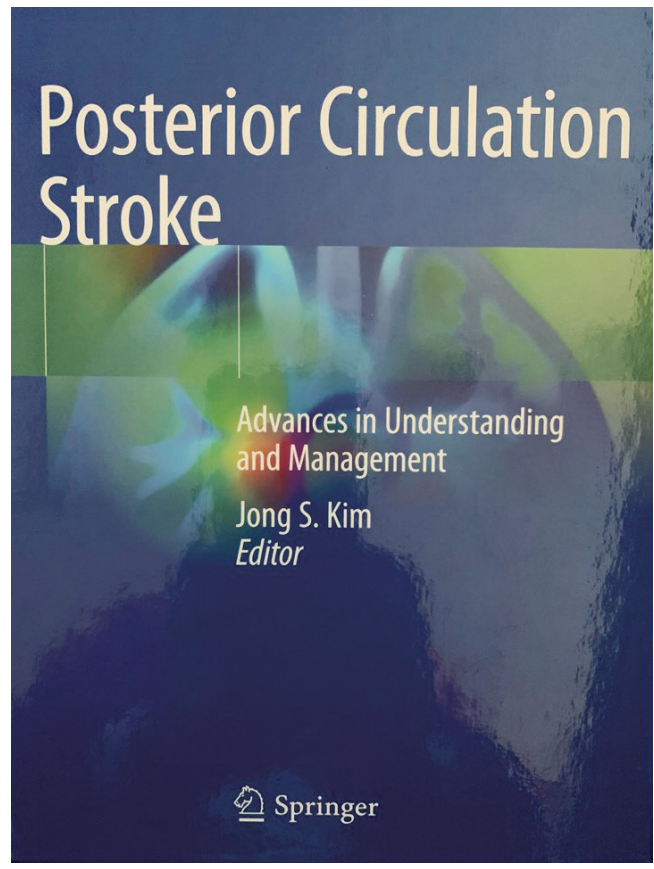

Figure 1. The book cover. brain is there such a busy series of highways, crossroads, and way stations so that even small lesions can create quite dramatic and specific effects, sometimes devastating. Speaking personally as one who saw clinical neurologists in action before the imaging era, there was no occasion more impressive than when a consultant neurologist was asked for an opinion in an open forum concerning a complex array of brainstem symptoms and signs, following which (usually he!) would then pronounce the location of the lesion within an anatomical millimetre. The attending audience would gasp in admiration; of course, no one could disagree given that the final evidence was usually lacking. However, this was not the case when opinions were given at one of the commonly held brain cutting sessions when the answer was revealed, sometimes dramatically by the final arbiter, the neuropathologist. Well do I recall these events at the Massachusetts General Hospital in Boston during my postgraduate studies when the final clinical opinion was given by $C$. Miller Fisher and the denouement by E.P. Richardson. The mantra that we learnt our neuroanatomy "stroke by stroke" was telling.

The advent of neuroimaging in the early 1970s, first with computed tomography and then magnetic resonance imaging changed everything. Lesion location became more obvious and, at first sight, much simpler. However, there was still consummate skill required in aligning the symptoms and signs with the imaging findings to make sure that these were, indeed, responsible for the phenotypic expression. At the same time, neuroimaging also created the opportunity for neurologists to redefine the classical brainstem syndromes and more fully explain the effects of stroke lesions of various sizes, shapes, and location. This is certainly evident in this volume where each chapter is written by current experts and world leaders in their field. 
In some ways posterior circulation stroke has been the poor cousin, particularly in terms of stroke treatment. Perhaps this has been, in part, because it has been more difficult to determine specific aetiologies compared to the anterior circulation. Imaging the ischemic penumbra has also been more difficult; hence, the benefits of various acute interventions have been harder to establish.

The book has a nice logical order to the chapters commencing with a delightful historical overview by Louis R. Caplan. There is no one who has contributed more to our understanding of the clinical features and pathophysiology of posterior circulation stroke than Caplan. His own book, Caplan's stroke: $a$ clinical approach, is a classic. He effortlessly takes us through the timelines of the description of disease of the posterior circulation from the Swiss pathologist and physician Johann Webster, through the work of the famous French physician Charles Foix at the Salpetriere Hospital in Paris, then to the seminal work of C. Miller Fisher and Raymond Adams in Boston, Wylie McKissock in London before moving on to describe modern imaging and treatment.

The first half of the book is taken up with this historical overview and then moving onto arterial anatomy and collaterals, epidemiology risk factors, and stroke mechanisms, brainstem and infarction syndromes, thalamic and posterior circulation artery syndromes. Even ocular, vestibular and otological syndromes and later haemorrhagic strokes have their time in the sun. The second half is then devoted to imaging diagnosis, treatments including medical interventions, thrombolysis and thrombectomy, angioplasty and stenting, and surgical intervention. To round the volume off nicely with beautiful symmetry, the final two chapters are devoted to uncommon diseases, outcomes, and prognosis.

As a clinical neurologist, I certainly enjoyed reading this book enormously. However, would also see it as being very accessible and a valuable reference source for medical students and other healthcare professionals with an interest in stroke. Indeed, this really is a book for the ages which should take its rightful place in the bookshelf of the pantheon of essential references on stroke.

Correspondence: Geoffrey A. Donnan

Department of Neurology, Melbourne Brain Centre, Royal Melbourne Hospital, University of Melbourne, 300 Grattan street, Parkville 3050, Melbourne, Australia Tel: +61-3-9342-4405

E-mail: geoffrey.donnan@unimelb.edu.au

https://orcid.org/0000-0001-6324-3403

Received: December 4, 2020

Revised: December 4, 2020

Accepted: December 4, 2020

The author has no financial conflicts of interest 